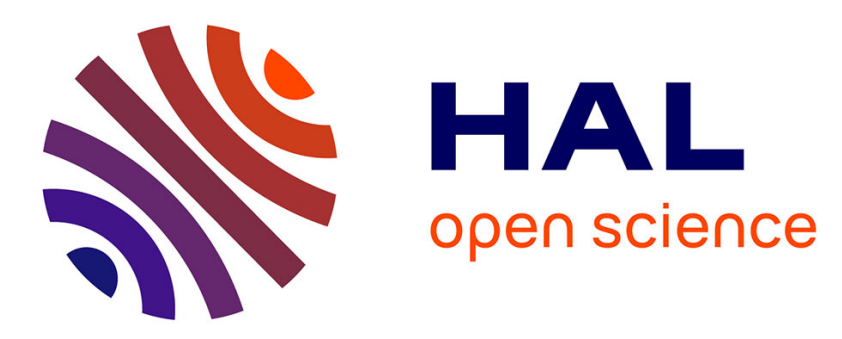

\title{
A Cognitive and Affective Architecture for Social Human-Robot Interaction
}

Wafa Johal, Damien Pellier, Carole Adam, Humbert Fiorino, Sylvie Pesty

\section{To cite this version:}

Wafa Johal, Damien Pellier, Carole Adam, Humbert Fiorino, Sylvie Pesty. A Cognitive and Affective Architecture for Social Human-Robot Interaction. 10th Annual ACM/IEEE International Conference on Human-Robot Interaction Extended Abstracts, Mar 2015, Portland, United States. 10.1145/2701973.2702006 . hal-01206483

\section{HAL Id: hal-01206483 \\ https://hal.science/hal-01206483}

Submitted on 15 Sep 2016

HAL is a multi-disciplinary open access archive for the deposit and dissemination of scientific research documents, whether they are published or not. The documents may come from teaching and research institutions in France or abroad, or from public or private research centers.
L'archive ouverte pluridisciplinaire HAL, est destinée au dépôt et à la diffusion de documents scientifiques de niveau recherche, publiés ou non, émanant des établissements d'enseignement et de recherche français ou étrangers, des laboratoires publics ou privés. 


\title{
A Cognitive and Affective Interaction-Oriented Architecture for Social Human-Robot Interaction
}

\author{
Wafa Johal, Damien Pellier, Carole Adam, Humbert Fiorino, Sylvie Pesty \\ Univ. Grenoble Alpes, LIG \\ F-38000 Grenoble, France \\ firstname.lastnamel@imag.fr
}

\begin{abstract}
Robots find new applications in our daily life where they interact more and more closely with their human user. Despite a long history of research, existing cognitive architectures are too generic and hence not tailored enough to meet specific needs of social HRI. In particular, interaction-oriented architectures require handling emotions, language, social norms, etc. In this paper, we present an overview of a Cognitive and Affective Interaction-Oriented Architecture for social human-robot interactions, called CAIO. This architecture is in the line of BDI (Belief, Desire, Intention) architecture that comes from action philosophy of Bratman. CAIO integrates complex emotions and planning technics. It aims to contribute to cognitive architectures for HRI by enabling the robot to reason on mental states (including emotions) of the interlocutors, and to act physically, emotionally and verbally.
\end{abstract}

\section{INTRODUCTION}

Robots and artificial agents with capacities of perception and action are more and more present in daily life. Nowadays, robots tend to be found in the developed world in domains such as search and rescue, space exploration, hospital care, daily life companion robots, etc. For all these new domains of applications, robots must have a closer interaction with the user. By closeness we mean that a robot must have to share not only the same physical space but also goals and beliefs to achieve a common task through their interactions. The study of interactions between humans and robots is thus fundamental to ensure the development of robotics and to devise robots capable of socially interacting intuitively and easily through speech, gestures, and facial expressions. Many applications involving robots exist, but the underlying robot's cognitive architecture is not always explicited. The development of a cognitive architecture for robots able to take into account the complexity of social human-robot interaction is a real challenge. It requires various features to be present in the robot architecture: emotions (non-verbal aspects of interaction), reac- tive and deliberative levels (fast (emotional) answer versus slower and more deliberate answer), explicit manipulation of mental states (to enable self-explanation).

Ever since the pioneer works on robot and cognitive architectures, e.g., 3, researchers have developed several architectures based on different underlying techniques, e.g., symbolic, connectionist or hybrid. For instance, some architectures are based on a set of generic and symbolic rules such as Soar [5] based on the unified theory of cognition, or ACT-R 2. Many of these architectures are based on the-mind-islike-a-computer analogy. In contrast, other techniques specify no such rules a priori and relies on emerging properties of processing units. Hybrid architectures combine both types of processing. CLARION [9] is an example of such architecture. In spite of the numerous contributions in the field of cognitive architectures, credible social interactions have not yet reach a reality. Therefore, it is necessary to research on different approaches to build cognitive architecture oriented for interaction able to deal emotion and social aspects of HRI.

\section{CAIO ARCHITECTURE}

The CAIO architecture (see figure 1) aims to contribute to cognitive architectures for HRI by: reasoning on five mental states called BIGRE (Beliefs, Ideals, Goals, Responsabilities, Emotions) of the interlocutors; and acting physically, emotionally and verbally.

The CAIO architecture consists in two loops : a deliberative loop used to reason on BIGRE mental states and produce plans of action, and a shorter reactive loop to immediately trigger emotions. Each loop takes as inputs the result of the multi-modal perception of the environment (the interaction with the human). During the deliberative loop, the deliberation module deduces the robot's communicative intentions based on its BIGRE mental states and the mental states of the interlocutor. Then, it selects the most appropriate one. The planning and scheduling module produces a plan to achieve the selected intention (i.e. a set of ordered actions, communicative acts and/or physical actions), and schedules the robot's next action. Finally the multimodal action renderer executes this scheduled action. Modules can provide feedback to each other: the planning module informs the deliberation module of the feasibility of the selected intention; the action renderer informs the planner of the success or failure of action performance. Simultaneously, during the reactive loop, the appraisal module uses the 
mental states to trigger the robot's complex emotions (regret, disappointment, guilt, reproach, moral satisfaction, admiration, rejoicing and gratitude) that can be directly expressed by the multi-modal action renderer via the corresponding expressive communicative acts. Both emotion and action are finally merged by the multi-modal action renderer to produce the appropriate interaction with the robot's available actuators. For instance, in the case of a verbal action, the action renderer module produces the facial expression that fits the emotion and utters the propositional content of the communicative acts.

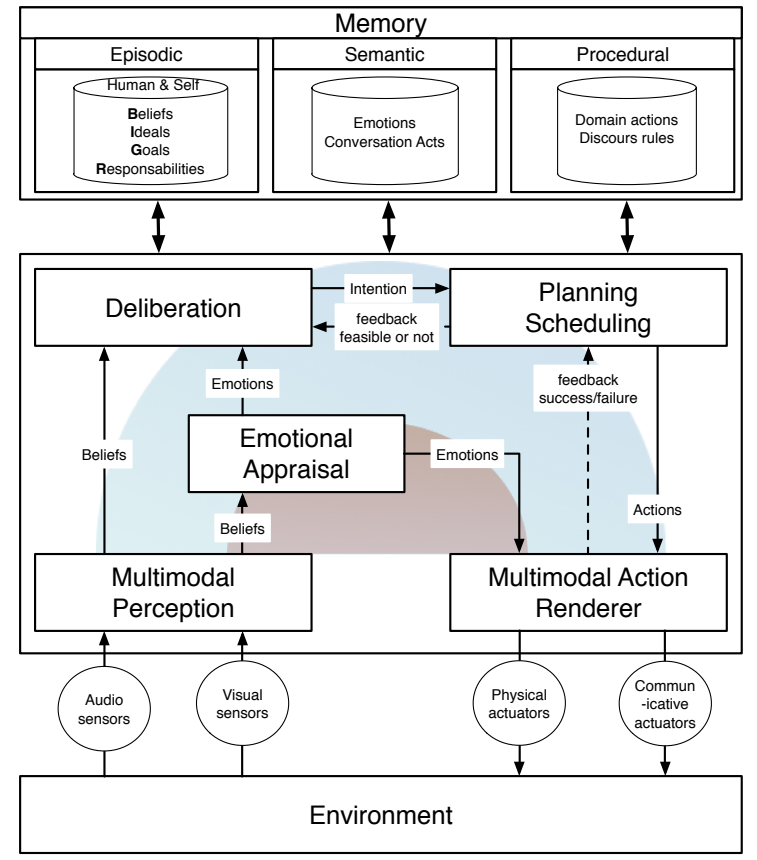

Figure 1: The CAIO architecture.

CAIO architecture is made up of 5 modules:

Multimodal perception module. The aim of the multimodal perception module is to merge multi-modal inputs such as natural language and facial expressions in order to generate beliefs on the user's mental states. This stimulus will then be evaluated by both emotional appraisal and deliberative modules.

Memory module. The robot's memory is divided into three parts in accordance with the state of the art. The episodic part contains BIGRE 4 based knowledge representations of self and human in interaction. The semantic part is composed by definition of emotions concepts and conversational acts. The procedural part deals with the domain action (how-to) and the discourse rules (i.e. when asked a question, one should reply).

Appraisal module. The appraisal module takes as input the robot's perceptions and mental states and triggers the complex emotions from their logical definition in terms of the mental states. See 8 for more details.

Deliberation module. The deliberation is the process that allows to decide the robot's communicative intentions, i.e., the goal to achieve. The CAIO architecture uses three kinds of communicative intentions: the emotional and obligation-based intentions useful to local dialogue regulation, and the global intention which defines the dialogue direction. The robot's communicative intention is selected via practical reasoning from its mental states and a set of priority rules. See 1, 7, for more details.

Planning module. The planning module 6$]$ is in charge of finding a way of achieving the selected communicative intention according to a plan-based approach of dialogue. The plans produced contain communicative acts and/or physical actions. The formalization of their preconditions and effects used the classical planning formalism PDDL (Planning Domain Description Language).

Multimodal Action Renderer. The last module of the CAIO architecture is the multi-modal action renderer. This module takes as input an action to be executed and the complex emotion computed by the appraisal module. The role of this module is to control the robot's actuators to executed the input action and to dynamically generate the facial expression to accompany the communicative acts achieving the communicative intention selected by the deliberation module.

\section{CONCLUSION}

In this paper, we have given an overview of a new affective and cognitive architecture for HRI, called CAIO. CAIO provides new contributions regarding the state of the art in cognitive architecture for companion robot. The two main ones lies in (1) its two loops : a deliberative loop used to reason on BIGRE mental states and produce plans of action, and a shorter reactive loop to immediately trigger emotions and (2) in the integration of emotions in HRI. We are conscious that this architecture has not achieved its final state and that some points need to be improved. Several improvements are under way. For instance, we are working on a learning module that could enable the robot to learn ontology that can the be used as new rules (procedural memory) and new concepts (semantic memory) by its reasoning process. We are also working on an experimental scenario to evaluate CAIO architecture involving children.

\section{REFERENCES}

[1] C. Adam. The emotions: from psychological theories to logical formalisation and implementation in a BDI agent. PhD thesis, INP Toulouse, July 2007.

[2] J. R. Anderson. Human symbol manipulation within an integrated cognitive architecture. Cognitive Science, 29(3):313-341, 2005.

[3] R. Brooks. A robust layered control system for a mobile robot. IEEE Journal of Robotics and Automation, 2(1):14-23, 1986.

[4] J. Guiraud, L. D., E. Lorini, S. Pesty, and J. Rivière. The face of emotions: a logical formalization of expressive acts. In $A A M A S^{\prime} 11$, pages 1031-1038, 2011.

[5] J. E. Laird. The Soar cognitive architecture. MIT Press, 2012.

[6] D. Pellier, H. Fiorino, and M. Métivier. A new approach for continual planning. In $A A M A S$, pages 
1115-1116, 2013.

[7] J. Riviere, C. Adam, and S. Pesty. A reasoning module to select ECA's communicative intention. In $I V A$, pages 447-454, 2012.

[8] J. Riviere, C. Adam, S. Pesty, C. Pelachaud,

N. Guiraud, D. Longin, and E. Lorini. Expressive multimodal conversational acts for saiba agents. In IVA, pages 316-323, 2011.

[9] R. Sun. A tutorial on clarion 5.0. Technical report, Cognitive Science Department, Rensselaer Polytechnic Institute, 2003. 